International Journal of Current Advanced Research

ISSN: O: 2319-6475, ISSN: P: 2319 - 6505, Impact Factor: SJIF: 5.995

Available Online at www.journalijcar.org

Volume 6; Issue 4; April 2017; Page No. 3484-3487

DOI: http://dx.doi.org/10.24327/ijcar.2017.3487.0299

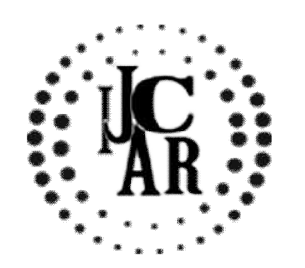

Review Article

\title{
ABSORPTION COEFFICIENTS OF FEW RESTORATIVE DENTAL MATERIALS
}

\author{
Rajasekhar E1*., Ramakrishnaiah N1., Disha Jain² and M.Chandra Sekhar² \\ ${ }^{1}$ Department of Physics, Rayalaseema University, Kurnool-518007, A.P \\ ${ }^{2}$ Department of Conservative Dentistry \& Endodontics, G.Pulla Reddy Dental College \& Hospital, \\ Kurnool-518007, A.P
}

\section{A R T I C L E I N F O}

\section{Article History:}

Received $8^{\text {th }}$ January, 2017

Received in revised form $16^{\text {th }}$ February, 2017

Accepted $9^{\text {th }}$ March, 2017

Published online $28^{\text {th }}$ April, 2017

\section{Key words:}

Absorption coefficient, half value layer, $\mathrm{pH}$ values, $\gamma$-source and restorative dental materials.

\begin{abstract}
A B S T R A C T
The change of intensity of the material can be explained in terms of absorption coefficient. The measurement of absorption coefficient, half value layer and $\mathrm{pH}$-values of few restorative dental materials were investigated using gamma radiation. Results show that composite resin has shown lowest absorption coefficient and Zinc Polycarboxylate has shown highest absorption coefficient values. As a result of low absorption coefficient, composite resin was considered a very good absorber and a good material for shielding gamma rays. A low absorption coefficient was desired as restorative materials used in the tooth. A high absorption coefficient was desirable where the material covers soft tissue. Descending order of absorption coefficient of the restorative dental cements were Zinc Polycarboxylate (ZPC), Zinc Oxide-Eugenol (ZO), Zinc phosphate (ZP), Silver amalgam (SA), Glass Ionomer (GI) and Composite resin (CR). Absorption coefficient can best be used in sorting the gamma radiation shielding abilities of dental materials.
\end{abstract}

Copyright $\subseteq 2017$ Rajasekhar $\boldsymbol{E}$ et al. This is an open access article distributed under the Creative Commons Attribution License, which permits unrestricted use, distribution, and reproduction in any medium, provided the original work is properly cited.

\section{INTRODUCTION}

For a scientific study of interaction of radiation with matter a proper characterization and measurement of penetration of gamma rays in the external medium is necessary. The study of interaction of gamma radiations with the materials of industrial, biological and commercial significance has become major area of interest in the field of radiation science. The study of absorption coefficient of various materials has been an important part of research work in radiation physics and chemistry. Hubbell has compiled an extensive data on absorption coefficients of gamma rays in some compound and mixtures of interest in the energy range of $1 \mathrm{keV}$ to $20 \mathrm{MeV}$. Radiations are usefully employed in various fields such as medicine, industry and agriculture [1][2][3][4]. A restoration is a material which substitutes the missing tooth structure and restores the form and function of the tooth. Temporary restorations are often required before the placement of a permanent restoration. Materials used for temporary restorations are expected to last for only a short period of time. They serve as an interim restoration while the pulp heals and till the permanent restoration can be fabricated and inserted. Examples of permanent restorative materials are direct filling gold, amalgam, composite resins, and glass ionomer cement.

*Corresponding author: Rajasekhar E

Department of Physics, Rayalaseema University, Kurnool518007, A.P
For many years, there has been a great interest in the establishment of specifications for dental materials on an international level. Two organizations, the Federation Dentaire International (FDI) and International Standards Organization (ISO) are working toward that goal. The FDI and ISO have the objective of developing international standards. The benefit of such specifications has been inestimable to the dental professionals. The dentist is provided with the criteria of selection that are impartial, reliable and assure uniformity. Different luting agents have been used in dentistry for more than a century. Today these materials can be categorized in two main classes according to ISO-standards: Dental acid-based cements consisting of zinc phosphate cements, polycarboxylate cements, glass ionomer cements, resin-modified glass ionomer cements and dental polymer-based luting materials such as composite resin [5]. Dental cements are commonly used clinically as a biomaterial development. Dental restorative materials used today include biomaterials such as resin composites, glass-ionomer cements and resin-modified glass-ionomer cements [6].

The measured intensity $I$ of transmitted through a layer of material with thickness $x$ and the incident intensity $I_{0}$ according to the inverse exponential power law that is usually referred to as Beer-Lambert's law:

$$
I(x)=I_{0} e^{-\mu x} \quad--------(1)
$$

Where, $\mu$ denoted absorption coefficient, $x$ is the thickness of the material. The attenuation factor of a material is obtained 
by the ratio of the emergent and incident radiation intensities $\frac{I}{I_{0}}$

The current investigation on the gamma ray absorption coefficients, half value layer and $\mathrm{pH}$ values of few restorative dental materials such as Composite resin (CR), Glass ionomer (GI), Silver amalgam (SA), Zinc Phosphate (ZP), Zinc oxideeugenol (ZO) and Zinc Polycarboxylate (ZPC) have been studied using $\mathrm{Co}^{60}$ gamma radioactive source with GeigurMuller (G.M) Counting System.

\section{MATERIALS AND METHODS}

\section{Collection of Dental Materials}

List of dental materials were shown in Table 1. Dental materials such as Composite resin (CR), Glass ionomer (GI), Silver amalgam (SA), Zinc Phosphate (ZP), Zinc oxideeugenol (ZO) and Zinc Polycarboxylate (ZPC) were collected from G.Pulla Reddy Dental College and Hospital Kurnool, Andhra Pradesh, India.

\section{Preparation of Sample}

The materials were mixed and handled according to manufacturers' directions. Three cylindrical specimens $13 \mathrm{~mm}$ diameter and $1.6 \mathrm{~mm}$ thick of each material were prepared in a mold and pressed between two microscope glass slides. Dental material was made in the form of pellet (absorber). The conventional glass ionomer cements (GICs) were allowed to set at room temperature for $10-15 \mathrm{~min}$.

Table 1 List of Dental Materials and Its Nature

\begin{tabular}{cccc}
\hline S.No. & $\begin{array}{c}\text { Name of the dental } \\
\text { materials }\end{array}$ & Code & Nature \\
\hline 1 & Composite resin & CR & Restorative materials \\
2 & Glass ionomer & GI & Restorative materials \\
3 & Silver amalgam & SA & Restorative materials \\
4 & Zinc Polycarboxylate & ZPC & Liners and bases \\
5 & Zinc phosphate & ZP & Liners and bases \\
6 & Zinc oxide-eugenol & ZOE & Liners and bases \\
\hline
\end{tabular}

\section{Experiment}

First we make the standard connections and arrangement between Geigur-Muller (G.M) Counting System, detector, absorber and source. Place a gamma $(\gamma)$ source cobalt-60 $\left({ }^{60} \mathrm{Co}\right)$ in the source tray at about $2 \mathrm{~cm}$ from the end window of the G.M tube. Set the G.M voltage at the operating voltage $(625 \mathrm{~V})$ of the GM tube. Place the absorber between end window detector and source holder containing absorbers of respective thickness. We took the reading for the period of 60 sec without any absorber and tabulated the experiment by recording the data stored for different thickness in the increasing order for the same period of $60 \mathrm{sec}$. Repeat the same steps as explained above for next absorber sets of dental material.

\section{Absorption Coefficient ( $\mu$ ) Procedure}

1. Place a $\gamma$ source in the source tray at about $2-4 \mathrm{~cm}$ from the end window of the G.M tube.

2. Set the G.M voltage at the operating voltage $625 \mathrm{~V}$ of the G.M tube.

3. Place the absorber from the absorber set and place it between the source and the tube and record the counts for $60 \mathrm{sec}$.

4. Replace the absorber with next one from the set taking care not to disturb.
5. Repeat the above step with thickness increasing and decreasing order.

6. Remove the source and absorbers and record the background counts for $60 \mathrm{sec}$.

When gamma radiation passes through matter it undergoes absorption primarily by Compton, Photoelectric \& Pair production interactions. The intensity of $\gamma$-rays passing through a medium is given by

$I(x)=I_{0} e^{-\mu x}$

Where,

$\mathrm{I}(\mathrm{o})=$ original intensity of beam (without an absorber)

$\mathrm{I}(\mathrm{x})=$ intensity through absorber of thickness $\mathrm{x}$.

$\mu=$ absorption coefficient of the medium $\left(\mathrm{cm}^{-1}\right)$.

Plot the graph of $\ln \left(\frac{I_{0}}{I}\right)$ Vs thickness of absorber. The slope graphs gives as absorption coefficient.

$\frac{\ln \left(\frac{I_{0}}{I}\right)}{X}=\mu$

Half Value Layer $(\mathrm{HVL})=\frac{\ln (2)}{\mu}$

\section{RESULTS}

The absorption coefficient, Half Value Layer (HVL) and $\mathrm{pH}-$ values were characteristics that can be used in restorative dental cements for primary and secondary use. Absorption coefficient can best be used in sorting the gamma radiation shielding abilities of dental materials. Dental materials are used widely in modern restorative dentistry because of their excellent harmony with natural teeth. The change of intensity of the material can be explained in terms of absorption coefficient of the materials. Results show that composite resin has shown lowest absorption coefficient and Zinc polycarboxylate has shown highest absorption coefficient values. Descending order of absorption coefficient of the dental cement materials were Zinc Polycarboxylate (ZPC), Zinc oxide-eugenol (ZOE), Zinc Phosphate (ZP), Silver amalgam (SA), Glass ionomer (GI) and Composite resin (CR). A small absorption coefficient indicates that the material is relatively transparent, while larger values indicate greater degrees of opacity. A low absorption coefficient was desired in restorative materials used in the tooth. A high absorption coefficient was desirable where the material covers soft tissue. The absorption coefficient is dependent upon the type of material and the energy of the incident radiation. Figure 1 shows absorption coefficients of different dental cement materials.

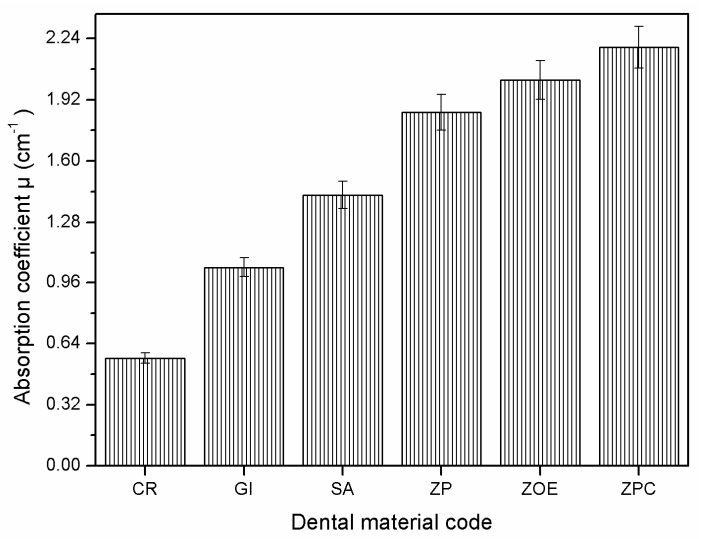

Fig 1 Absorption coefficient $(\mu) \mathrm{cm}^{-1}$ of dental materials 
As a result of low absorption coefficient, composite resin is considered a very good absorber and a good material for protecting gamma rays. The half value layer (HVL) is the thickness of a material required to reduce the intensity of radiation at a point to one half of its original intensity. Figure 2 shows that Calculation of Half Value Layer (HVL) of various dental materials used for the experiment. It can be seen that composite resin has the highest half value layer and Zinc polycarboxylate has the lowest half value layer. It means that a lesser thickness of composite resin will be required to attenuate gamma radiation to half its original intensity, when compared with other investigated dental materials. The lowest half value layer of material has the highest attenuation ability, this implies a bad absorber of radiation and highest half value layer of composite resin has the lowest attenuation ability, this implies the material is a good absorber of radiation. Descending order of half value layer of the dental cement materials were Composite resin (CR), Glass Ionomer (GI), Silver amalgam (SA), Zinc phosphate (ZP), Zinc oxide eugenol (ZOE) and Zinc polycarboxylate (ZPC). From the above results, absorption coefficient and half value layers are characteristics that can best be used in shielding abilities of materials.

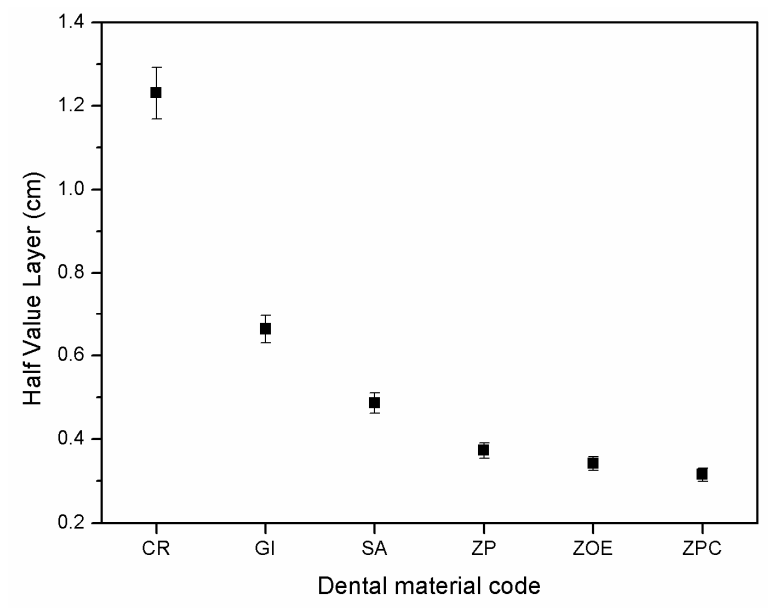

Fig 2 Half Value Layer vs. Dental material code

The $\mathrm{pH}$ profiles for all tested materials at $24 \mathrm{~h}$ were given in Figure 3. Metal is a good conductor of cold and heat to the nerve of the tooth. Commercial dental cements used for interim restorations. Adverse affect of glass ionomer cement on living tissues are negligible. One-way ANOVA showed significant differences $(p<0.05)$ of all tested materials. Polymers of resin composites should not be degraded in an oral environment, in which the $\mathrm{pH}$ changes dynamically in an aqueous medium. The $\mathrm{pH}$ varies in the oral cavity according to the oral environment and tooth surface conditions. Toothcolored restorative dental materials are attractive because of the harmonious match with the host teeth by satisfying the aesthetic requirements of a range of users. The restored dental materials are influenced by the dynamic changes of the environment in the oral cavity. A low $\mathrm{pH}$ carbonated can influence the properties of tooth coloured adhesive materials used to restore cervical lesion. The tested specimens were significantly different between the $\mathrm{pH}$ solutions. The long term mechanical stability of self adhesive composite resin plays a crucial role in clinical success of indirect restorations. According to these findings, when using self adhesive resin luting agents and auto mix products with high $\mathrm{pH}$ neutralization behaviour should be favoured by clinicians.
Inflammatory response of pulp towards glass ionomer cement due to its initial $\mathrm{pH}$ of 0.9 to 1.6 resolves within 21 to 30 days.

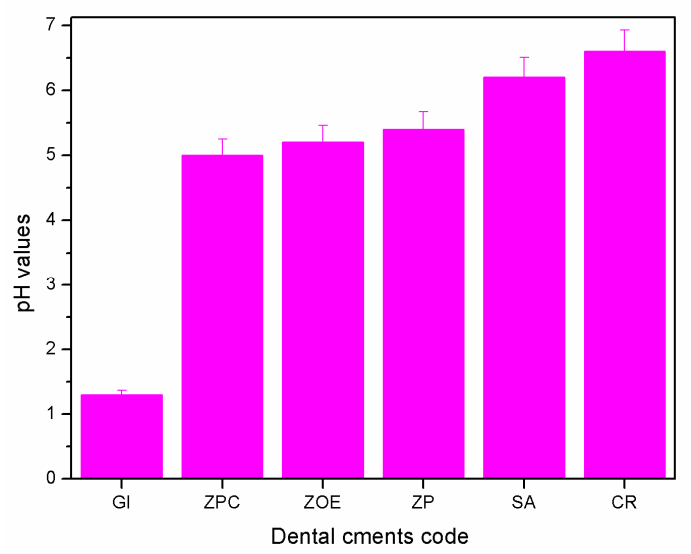

Fig 3 Dental cements vs. pH values

\section{DISCUSSION}

Gamma rays were produced by many radioactive substances. They can also be found in nuclear reactors and in cosmic radiation. There are many useful applications of gamma ray such as radiotherapy, medical tracer and sterilization. When gamma rays are absorbed by a living organism, they may cause vital hazards. Therefore, it is necessary to find out some substances that can be effectively absorb and block $\gamma$-rays. Dental materials are used widely in modern restorative dentistry because of their excellent harmony with natural teeth. A preferable material is expected to have low gamma ray absorption coefficient in orders that a small thickness will produce significant reduction in intensity. The Preferable order of restoration dental cements based on absorption coefficient and half value layer were Composite resin (CR), Glass ionomer (GI), Silver amalgam (SA), Zinc phosphate (ZP), Zinc oxide-eugenol (ZOE) and Zinc Polycarboxylate (ZPC). From the above results, absorption coefficient and half value layers are characteristics that can be used in sorting the gamma radiation protective abilities of dental materials.

Glass ionomer cement is a dental restorative material used in dentistry for dental fillings and luting cements. It is commonly used as an orthodontic bracket adhesive, either as glass ionomer or glass ionomer based cement. Among them, resin composites are the most popular restorative material. The advantage of resin composites for restoration would be a colour match to the adjacent teeth with a wide range of shade options as well as agreeable mechanical properties. Previous studies reported that restorative resin composites are discoloured when exposed to the diverse oral environment. The discoloration of resin composites might be mediated by water. Chemical degradation can occur if resin composite absorbs water and other colorants because water is an excellent solvent and cheap availability in nature. Clinically, this can lead to a loss of restoration contour, as well as an increase in surface roughness and discoloration [7][8][9][10][11].

Amalgam is a combination of metals that has been the most popular and effective filling material used in dentistry for the last 150 years. It is sometimes called silver amalgam. Amalgam actually consists of a combination of $\mathrm{Hg}$ and 
metals. These include silver, mercury, tin and copper. Amalgam is less costly than other materials.

According to previous studies, a lower $\mathrm{pH}$ had been shown to have an adverse effect on the wear resistance of resin composites. In addition, highly alkaline solutions have been shown to accelerate hydrolysis and produce surface micro structural damage. According to Poggio et al., cola had the lowest $\mathrm{pH}$ (2.55) and might damage the surface integrity of resin composites [12]. Buchalla et al. reported that storage in acidic solutions had very little effect on resin based luting cements [13]. Many hydroxyl ions were presented in solutions at neutral $\mathrm{pH}$ or low $\mathrm{pH}$. The strong influence of the alkaline medium on the composite properties was attributed to their interactions with $\mathrm{OH}$ ions during the hydrolysis process.

Dental cements are the most widely used materials for temporary restorative purposes. These consist of a powder and a liquid when mixed produces a plastic mass, which is inserted into the cavity and allowed to set. Dental materials are of comparatively low strength, but they are used extensively in dentistry. They possess so many desirable characteristics that they are used in $40-60 \%$ of all restorations. These are used for temporary and permanent restorations as root canal sealants, as cavity liners and for pulp capping. The mixed cement should never be applied directly onto dentine near to pulp or onto exposed pulp. The cement must be applied to the dry tooth surface and must set without contact to moisture. Clean instruments and glass plate with water before the cement sets. Zinc polycarboxylate cement can be dissolved using a cement solvent after setting. Solubility and disintegration can be reduced by proper manipulation, minimizing the exposure of the cement to the oral environment and protecting of the cement during setting and the initial 24 hour period.

\section{CONCLUSION}

Dental materials were used widely in modern restorative dentistry because of their excellent harmony with natural teeth. Absorption coefficient can best be used in sorting the gamma radiation shielding abilities of the restorative materials.

Long term studies also demonstrate that amalgam can be safely handled by dentists and other dental health care workers in occupational settings. Findings from scientific research and major national and international health organizations support the use of dental amalgam as a safe and effective restorative material. The preferable order of restoration dental cements was Composite resin (CR), Glass ionomer (GI) and Silver amalgam (SA). Among them, resin composites are the most popular restorative materials. Finally we can conclude that Statistical significant differences among material properties of the tested materials. These differences may anticipate in vivo variations on long term clinical success of restorations.

\section{Acknowledgement}

The authors pleased to extend their gratitude to Dr.Y.MURALIDHAR REDDY Principal and Staff of G.Pulla Reddy Dental College \& Hospital, Kurnool, Andhra Pradesh for their constant support \& coordination.

\section{References}

1. Hubbell J.H. Photon mass attenuation and energy absorption coefficients from $1 \mathrm{keV}$ to $20 \mathrm{MeV}$; Appli. Radiat. Isot 1982; 33: 1269.

2. Hubbell J.H. and Seltzer S.M. Tables of X-ray mass attenuation coefficient and mass energy absorption coefficients $1 \mathrm{keV}$ to $230 \mathrm{MeV}$ for elements $\mathrm{z}=1$ to 92 and 48 additional substances of dosimetric interest. 1995; NISTIR-5632.

3. Singh Jarnail, Singh Karamjit, Mudahar S. and Kulwant S. Gamma ray attenuation studies in Telurite glasses, National Symposia on radiation Physics,2003; 15:36-39.

4. Chaudhari L.M. and Nathuram R., Absorption coefficient of polymers (Polyvinyl Alcohol) by using gamma energy of $0.39 \mathrm{MeV}$, Bulg. $J$. Phys.,2010;37:232-240.

5. Ferracane JL, Stansbury JW, Burke FJ. Self-adhesive resin cements-chemistry, properties and clinical considerations. J Oral Rehabil,201; 38:295-314.

6. Glasspoole E, Erickson R, Davidson C. A fluoride releasing composite for dental applications. Dent Mater, 200; 17: 127-33.

7. Klingshirn C. "ZnO: Material, Physics and Applications". ChemPhysChem.2007; 8(6):782-803.

8. Kenneth J. Anusavice, Chiayi Shen, H. Ralph Rawls. Phillips' Science of Dental Materials $12^{\text {th }}$ Edition,2016.

9. Prakki A, Cilli R, Mondelli RF, Kalachandra S, Pereira JC. Influence of $\mathrm{pH}$ environment on polymer based dental material properties. J Dent, 2005; 33:91-98.

10. Turssi CP, Hara AT, Serra MC, Rodrigues AL Jr. Effect of storage media upon the surface micromorphology of resin-based restorative materials. J Oral Rehabil, 2002; 29:864-871.

11. Correr G.M, Bruschi Alonso R.C, Baratto-Filho F, Correr-Sobrinho L, Sinhoreti M.A, Puppin-Rontani R.M. Invitro long-term degradation of aesthetic restorative materials in food-simulating media. Acta Odontol Scand, 2012;70:101-108.

12. Poggio C, Dagna A, Chiesa M, Colombo M, Scribante A. Surface roughness of flowable resin composites eroded by acidic and alcoholic drinks. $J$ Conserv Dent,2012; 15: 137-140.

13. Buchalla W, Attin T, Hellwig E. Brushing abrasion of luting cements under neutral and acidic conditions. Oper Dent,2000; 25:482-487.

\section{How to cite this article:}

Rajasekhar E et al (2017) ' Absorption Coefficients Of Few Restorative Dental Materials', International Journal of Current Advanced Research, 06(04), pp. 3484-3487.

DOI: http://dx.doi.org/10.24327/ijcar.2017.3487.0299 